\title{
Temporal Subordination and the English Perfect
}

\author{
Beverly Spejewski \\ University of Pennsylvania
}

\section{Introduction}

Researchers have long puzzled at restrictions on adverbial use with the present perfect, which don't seeem to apply to the simple past or past perfect:

(1) a. Jake has watered the garden $\{$ today/recently/*yesterday/*on Sunday $\}$.

b. Jake watered the garden $\{$ today/recently/yesterday/on Sunday\}.

c. Jake had watered the garden $\{$ that day/recently/the day before/on Sunday .

In attempting to explain the contrast between present perfect and simple past, and between present perfect and past perfect, various claims have been made about the structural relations introduced by the present perfect, which are special to that tense-aspect form. For instance, extended-now theories (Bennett \& Partee (1978), McCoard (1978), Vlach (1993)) propose that the present perfect introduces a timespan that contains the event and either runs up to or includes now. Any adverbial modifier must be able to modify this extended-now timespan. Current-relevance theories (Twaddell (1968), Comrie (1976), Moens \& Steedman (1988)) claim that the event must have some relevance to now in order for the present perfect to be used. Klein's (1992) p-definiteness constraint prohibits having both "topic time" and "situation time" assigned to independent known timepoints; the present perfect implicitly introduces now as a timepoint for topic time, and so no other known timepoint can be introduced for the situation time in which the event occurs.

Most analyses of adverbial use with the present perfect rely on non-compositional interpretations of tense and aspect; many do not relate the claims made for this particular construction to other elements of the language. However, I will show that the perfect can be cast as a specific case of a very general temporal construction, called "temporal subordination", and that in analyzing it this way, elements of the above analyses of the perfect will fall out naturally, as will the adverbial constraints illustrated in (2). In addition, a set of pragmatic effects on adverbial modification from Katz \& Spe jewski (1994) can be explained:

(2) a. My house has blown up \{??today/??this year\}.

b. ??Bill Clinton has been president.

c. Wade has walked the dog \{today/??this morning\}. —if he usually walks her some other time of day

d. Has Kay paid her bills $\{$ this month/??this week\}? —if she has monthly but not weekly bills 
Further data, involving the relation of events in subsequent sentences or clauses to those in the perfect sentence, is also predicted by the analysis.

I assume that the similarities between the simple past and the past perfect are due to the ambiguity of the past perfect form between a past-of-perfect and a past-of-past reading (after McCawley (1973), 259-268). The distinction can be illustrated by (3), where (3a) indicates a time in the non-ad jacent past (past-of-past reading) and (3b) is a past-of-perfect, indicating a timespan connected to the past reference time. There are also situations in which the past perfect can be used felicitously, but the present perfect can not or gives a different meaning (4) and (5). The majority of this paper will concentrate on the present perfect, since it is not ambiguous in this way; however, the past-of-perfect reading can be handled by the perfect analysis laid out in this paper.

(3) a. Jake had not watered the garden the day before.

b. Jake had not watered the garden since the day before.

(4) a. Jake regretted the events of the night before. He had called his boss a jerk and deleted some computer files.

b. Jake regrets the events of last night. ??He has called his boss a jerk and deleted some computer files.

(5) a. How had you broken your nose?

b. ??How have you broken your nose? (both from Michaelis, 1994)

In the next section, I will briefly lay out a general theory of temporal relations, which I refer to as a theory of temporal subordination. In section 3, I will show how the perfect can be accommodated by this theory and the adverbial constraints accounted for, and in section $4 \mathrm{I}$ will show how discourse data is predicted by the theory.

\section{The Temporal Subordination Theory}

The semantic theory of temporal subordination, as laid out in Spejewski \& Carlson (1992) and Spejewski (1994), proposes that there are two general kinds of temporal relation that can hold between two eventualities. The relation we call temporal subordination indicates that two eventualities occur at approximately the same time, and that there is some import to this temporal proximity. The other relation, temporal coordination, indicates that the events either occur in distinct time periods, or that there is no import to their occurring simultaneously.

\subsection{The two kinds of relation}

The temporal subordination relation incorporates a notion of contingency discussed by Moens \& Steedman (1988). Contingency is a perceived dependence between two 
eventualities, such as that found between causal and resultant events, between an event and a sub-event, or between the two events in a when-sentence. In these kinds of cases, either there is an apparently intrinsic dependence between the events, as with sub-events, in which case a hearer can usually identify the dependence without any overt linguistic cues, or else a speaker chooses to convey a perceived dependence by using overt linguistic cues, such as when. Temporal subordination applies to a subset of contingency cases: namely those where there is temporal dependence, such that one event occurs within the time introduced for another event. Typical examples of subordination are events related by event decomposition, when, or sentence-final then, or non-discourse-initial states.

Contingency is an asymmetric relation: it indicates that one event occurs within the time set up by another time. This can be represented using an asymmetrical temporal structure. I follow many researchers (e.g. Partee (1984), Hinrichs (1986), Kamp \& Reyle (1994)) in assuming that when a discourse is processed, each event or state will be related to a reference time. The reference time for an event will contain the event, and may extend beyond the event. The reference time for a state will be contained within the time of the state, and the state may hold beyond its reference time. The reference times of a pair of eventualities can be related in either a symmetric or asymmetric way, as we will see, according to whether contingency is perceived.

What is the reference time? In this work, it is a discourse object which represents a hearer's interpretation of the time during which the event is likely to have occurred, given the context of the discourse, or a time throughout which the state is likely to have held, given the discourse. For an event, the reference time must be a time that can contain the entire event; for a state, the reference time may be a subinterval of the time the state holds, and it will be that portion which is relevant to the discourse. Because the reference time reflects the hearer's interpretation, it is dynamic, so the specifications of the reference time may change as the discourse proceeds, and the boundaries typically will not be fixed or even specified. In interpreting a discourse, one typically does not have knowledge of the exact times that events occurred, or even the exact relative timings of events (e.g. that event 2 occurred three minutes after event l). Rather, we tend to have a sense of the relative relations among eventualities, where each event could have occurred within some vaguely-specified period of time. This period of time we refer to as the reference time. A hearer may use world knowledge (for instance, how long a particular event typically takes), discourse knowledge (such as why two events would be mentioned in sequence), or linguistic knowledge (understanding of temporal terms) in setting up the reference times.

Once a reference time is created, an event marker is inserted within it, or it is inserted within a state interval. We can represent this diagrammatically as in (6), so that if a temporal diagram is considered to be a tree (technically a graph), the daughter relation indicates that the daughter is contained within the parent. 
(6)<smiles>[R]S[14CH2][14CH2][14CH3]</smiles>

From the representation of the eventuality, we move to representing temporal relations among eventualities. Temporal subordination was described as indicating a dependence in which the subordinate event is contained within the time set up by the independent event. This is easy to illustrate with our tree-style notation by showing that the reference time $R_{2}$ for the subordinate event $e_{2}$ is contained in the reference time $R_{1}$ of event $e_{1}$ :

(7)<smiles>[Z20][R]Cl</smiles>

Notice that this structure does not require that the events themselves be overlapping; it simply says that the second event is constrained to be within the time introduced for the first event. The reason for this is illustrated by some when sentences from Ritchie (1979) and Moens \& Steedman (1988):

(8) When they built the fifth bridge,...
a. ... they took several bids.
b. ... they used the best materials.
c. ... they had a gala opening.

Here there is a clear linguistic marker (when) indicating that two events are to be interpreted with temporal contingency, but we can understand the main-clause event as occurring before, during, or after the when-clause event. Moens \& Steedman explain this effect by saying that this construction allows the events to introduce preparatory and consequent stages. The reference time in the temporal subordination approach could be considered as a vehicle through which this is possible.

The representation in (7) is equivalent to a Discourse Representation Theory (DRT) structure (Kamp \& Reyle (1993)), and can be implemented with the same model. The set below is a simplified set of predications that could represent the subordination structure for sentence (8c). Using the tree-type diagrams simply clarifies the relationship structure. 
(9) $R_{1} \subseteq R_{0}$

$e_{1}$ : Town build bridge

$\epsilon_{1} \subseteq R_{1}$

$R_{2} \subseteq R_{1}$

$\epsilon_{2}$ : Town have gala opening

$\epsilon_{2} \subseteq R_{2}$

In order to represent the coordination relation, I will first introduce one more assumption: that the discourse itself introduces a reference time, which serves as the overall timespan for the events of the discourse to be contained within. This global reference time is represented as $R_{0}$. Now, the coordination relation, as in (10) below, indicates that there is temporal independence between two eventualities, and this can be represented as in (11), where each reference time is inserted directly within $R_{0}$. This set of relations can also be represented in DRT formulas.

(10) Jimmy filled a bucket with water. Then he dumped it on his sister's head.

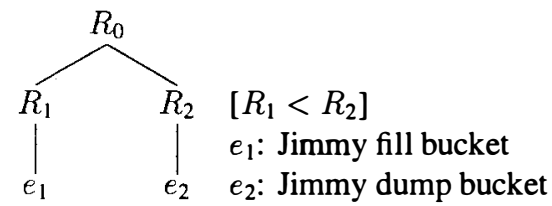

The relations work just the same for states as they do for events, with the only difference being that states will surround their reference times, rather than being contained within them. Both kinds of relation specify the relationships among reference times, not eventualities, and so the general relations can be specified in the same way, independent of the type of eventuality.

\subsection{The temporal structure of discourse}

Armed with the two temporal relations, subordination and coordination, we can represent entire discourses and also make predictions about the future structure of the discourse. To get a feel for what a temporal tree for a discourse could look like, we'll look at a short discourse:

(12) Edmond did a beautiful job of landscaping the yard $\left(e_{1}\right)$. He put fruit trees in the back yard $\left(e_{2}\right)$. In the front yard he made a large flower garden $\left(e_{3}\right)$. In it he planted both cultivated and wild roses $\left(e_{4}\right)$, and then he installed a fountain in the middle $\left(e_{5}\right)$. In the side yard was a wonderful herb garden $\left(s_{6}\right)$.

The first event is an overall event which the rest of the discourse describes in more detail, and so all of $e_{2}-s_{6}$ will be subordinate to $e_{1}$. (Recall that states surround their reference times.) However, there is also a further breakdown of $e_{3}$, with the next two events directly subordinate to it. The diagram for this discourse would be: 
(13)

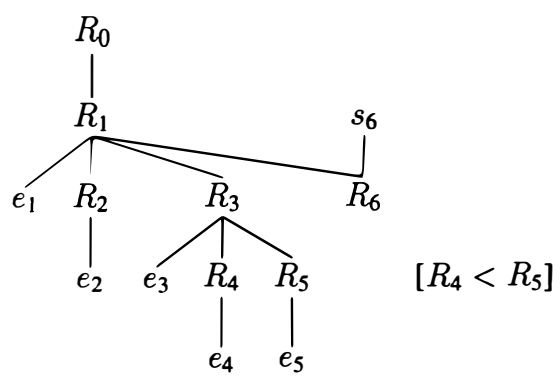

There is one special formula indicating the actual temporal ordering of two reference times: $R_{4}<R_{5}$. This is included because of the word then relating $e_{4}$ and $e_{5}$. For the rest of the event pairings, we have neither linguistic information nor world knowledge to indicate what order any of the events were performed, so there are no other ordering formulas. However, we do have world-knowledge information indicating subordination/coordination distinctions.

This example illustrates the way in which combinations of coordination and subordination, along with optional ordering formulas, can represent the temporal structure of an entire discourse. Any number of eventualities can be coordinated together or subordinated to an eventuality, and an eventuality may be in different relations with different eventualities. For instance, $e_{4}$ is subordinate to $e_{3}$ but coordinated with $e_{5}$.

Eventualities (and their reference times) are incorporated into the tree left-toright as they appear in the discourse, and not as they occur temporally. This manner of incorporation makes it very easy to make predictions about how a new eventuality may be related to existing ones. We can borrow the right-frontier idea from rhetorical relations (Polanyi (1988), Webber (1991), Lascarides \& Asher (1991,1993)), which claims that when a structure like our temporal trees is built for discourse relations, a new node may only be added as the daughter of a node that is on the right frontier. The right frontier in our case includes any reference time which is the rightmost reference time in the tree at its structural level. This turns out to be equivalent to any reference time which has not had a subsequent reference time coordinated with it or with any of its ancestors (other than the global reference time). In diagram (13), nodes $R_{0}, R_{1}$, and $R_{6}$ are currently available as insertion sites. This means that the next eventuality added to this discourse may be incorporated as a daughter of any one of these reference times. The next eventuality could thus elaborate on $s_{6}$ or $\epsilon_{1}$, or it could begin a new independent temporal line as a daughter of $R_{0}$. The notion of insertion sites will be important in section 4 for predicting what kinds of discourse continuations are possible after an eventuality has been introduced in the perfect. 


\subsection{Temporal adverbs}

Since we will be analyzing the perfect with respect to temporal adverbial use, let us now look at how adverbials are interpreted in this theory, following Spe jewski (1994). There are three ways that temporal adverbials are incorporated into this analysis, according the kind of adverbial. A frame adverbial, such as yesterday, next month, or within the hour, indicates a timespan that contains one of the available reference times in the discourse. A clause such as (14a) indicates that the reference time for the event [I take my niece to the playground] is contained within yesterday. This is represented in (14b):

(a) Yesterday, I took my niece to the playground.

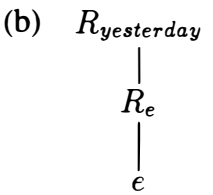

The representation of frame adverbials allows for the adverbial reference time to be available as a subsequent insertion site. This predicts that the next eventuality introduced in the discourse could also occur within that same time period specified by the adverbial, and this is perfectly appropriate.

Durative adverbials, such as for an hour or from midnight til two, specify how long an eventuality lasted. I follow Binnick (1991) in treating duratives as specifying a feature of the event rather than the reference time. Durative adverbials do not seem to introduce a new site for future events to be located, and so they do not introduce a new reference time. Instead, they introduce a predication on the event, as shown in (15):

$$
\begin{array}{ll}
\left.\right|_{e_{1}} ^{R_{0}} & \\
R_{1} & \epsilon_{1}: \text { Lizzy skate } \\
& \text { oneur }\left(e_{1}\right)
\end{array}
$$

In a few cases, it is clear that an adverbial is not meant to locate the eventuality in a specific temporal location, but rather it may be considered a modifier on the event type itself. These are cases of indefinite adverbials, where it doesn't matter which particular date the event occurred on, but only a particular property of when the event occurred, such as the following example, similar to one in Klein (1992):

(16) Lucy was criticized by her pastor because she worked on (a) Sunday.

Here the adverbial is part of the event itself, so the event in the because clause is [Lucy work on a Sunday]. 
Summarizing the main points of the theory of temporal subordination:

1) There are two temporal relations: subordination and coordination.

2) If $a$ is subordinate to $b$, then $a$ 's reference time is within $b$ 's reference time.

3) If $a$ is coordinated with $b$, then $a$ 's reference time is inserted as a sister of $b$ 's reference time.

4) Temporal orderings among reference times may be specified.

5) A new node may be inserted as the daughter of any reference time on the right frontier of the temporal tree for the discourse.

6) Frame adverbial reference times serve as parents of other reference nodes.

\section{Temporal Subordination and the Perfect}

\subsection{Analysis of the Perfect}

This basic theory of temporal relations applies fairly easily to the case of the perfect in English, handling both adverbial use and the semantics of the aspect, as well as making predictions about the effect that the perfect has on the introduction of subsequent eventualities in the discourse.

I propose to analyze the perfect as a case of temporal subordination within the clause, rather than between different clauses. Let me introduce the idea by discussing informally what the effect of the perfect is. Years of research on the present perfect has made it clear that there is some dependence relation (current relevance) indicated by the present perfect between the time of utterance and the time of the eventuality introduced, and also that these times are temporally related (extended-now). This gives us temporal proximity and contingency, the two notions we need in order to identify temporal subordination. Analyses based on Reichenbach (1947) also give us a three-time relation. All these pieces are part of temporal subordination. Looking at the past perfect makes it especially clear that the event introduced in the perfect is subordinate to an already-established time. With the past perfect, a temporal reference has been set up independently beforehand, and the new eventuality is located only with respect to that time.

The representation of the perfect is fairly straightforward in the system, if we add one formula as the special contribution of the perfect. Taking the general scheme of temporal subordination, the past perfect could be represented as in (17), where $\epsilon_{p r \epsilon v}$ is a previously-introduced event, whose reference time serves as the location for the event $c_{\text {I }}$ introduced by the past perfect. The past perfect can be represented by either of the following diagrams, depending whether you prefer to follow the standard representation or to incorporate temporal iconicity. In this case, both items are introduced at once, so there is no necessary order to their inclusion, and some people may find it easier to think about the temporal relations by referring to diagram (b). 
(17)

(a)

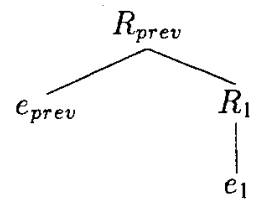

(b)

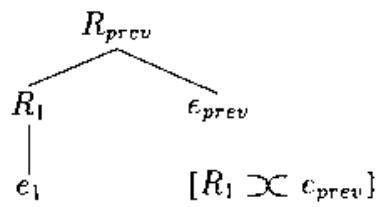

The symbol $\chi$ means "abuts" (from Kamp \& Reyle (1993). The ordering formula $\left[R_{1} \propto e_{\text {prev }}\right]$ is added to the general structure of subordination as the special contribution of the English perfect. This specification will account for some of the constraints on adverbial use, and also part of the semantics of the perfect. Notice that the formula specifying the abutting does not add any new structure to the representation of discourse. The representation for the perfect relies on structure that has been established for the overall theory, which is meant to handle many different kinds of cases. The kind of information that is added by the perfect is exactly the same kind of information that is added by a temporal modifier such as then. Then picks out the coordination structure, rather than the subordination structure, and adds its own contribution that the new reference time come after the one it is coordinated with. The analysis of the perfect here exactly follows the analysis of other temporal specifiers under this theory. No new theoretical constructs are needed in order to handle this aspect. I believe this is fairly unique among analyses of the perfect. It will be easier to understand the importance of the formula as we go along, so will we look first at the general analysis.

With the present perfect, the event is subordinated to now, or the speech event. This is similar to Vlach's "speech situation", which includes relevant properties of the speech time. The reference time introduced by now, which I have labelled as $R_{n o w}$, is a contextually-determined time that contains now. $R_{\text {now }}$ is similar to some versions of the "extended-now" as a timespan that includes now and also contains the event (Bennett \& Partee (1978), McCoard (1978)). Vlach (1993)) also allows for a time which contains the event and extends all the way up to now without containing it. With the constraint we have added that $R_{1}$ abut now for the present perfect, $R_{\mathrm{l}}$ instantiates this notion.

(a)

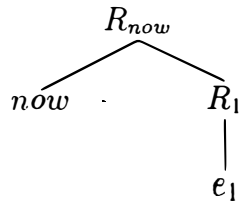

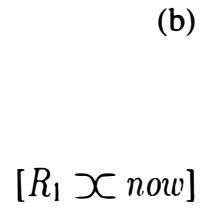

(b)

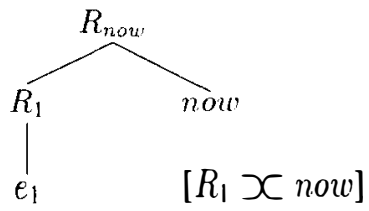

\subsection{Adverbials and Semantics}

With the general structure identified for the perfect, we can look at the way adverbials are constrained by this analysis. Recall that under the theory of temporal subordination, frame adverbials surround a reference time. With the perfect, we have two reference times introduced, providing two possible locations for adverbial attachment. But each reference time has constraints built into it, and any adverbial 
that modifies that time must accord with the constraints.

Containing $R_{\text {naw }}$ Any adverbial that contains the reference node $R_{\text {now }}$ will also have to contain now, since $R_{\text {now }}$ contains now. There are a number of adverbials that can contain now, if they are uttered at appropriate times. These include today, this moming, now, this year, and so forth. All of these adverbial are acceptable with the present perfect, and their use is represented in (19):

(a)

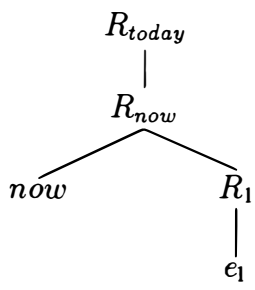

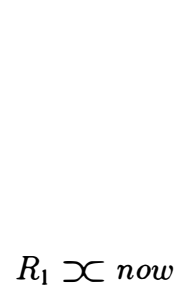

(b)

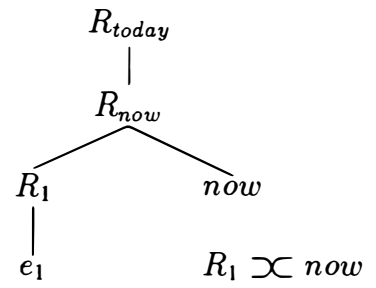

Containing $R_{e}$ If an adverbial is to contain $R_{e}$ instead, then it must match the constraints on $R_{e}$, which are that they contain the event and also abut now. Any adverbial time containing the event and not now must abut now. (If it contained now, then it would also contain $R_{\text {now. }}$.) Any adverbial defined as abutting now (or the established past referent, for the past perfect) can surround $R_{e}$, and is acceptable with the present perfect. This class of adverbials includes recently, just, ever, never, not yet, before (now), since Thursday, in the last hour and so on, and their use is represented in (20):

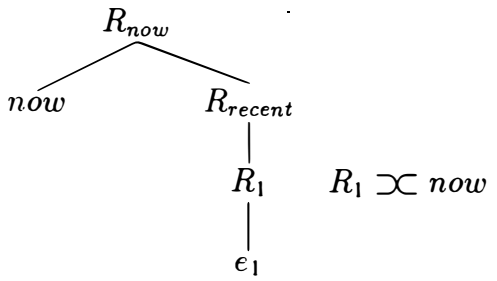

(b)

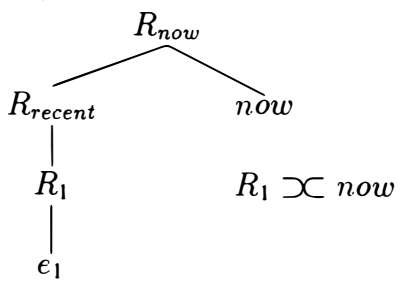

Unacceptable adverbials Now we can turn to an adverb that is not acceptable with the present perfect, and see how this analysis rules it out. Taking an adverb like yesterday, we know that it is defined as abutting the day the day containing now or speech time, but crucially not as abutting or containing now itself. Of the two candidate reference times, only $R_{1}$ does not contain now. But $R_{1}$ is constrained to abut now, and not the day containing now. The meaning of yesterday does not allow it to abut now, and so it cannot contain $R_{1}$. The same argument holds for any adverbial that picks out a specific time before now, such as at noon, on Thursday, before yesterday, or this moming if it is no longer morning. An adverbial like this past week will be acceptable or not, according to whether an individual allows the phrase to mean the seven days prior to now or only a calendar week.

Some adverbials that do not work with the perfect may be very similar to ones that do, except that they differ in whether they include the discourse-established 
time marker in their meaning, or whether they abut the marker. An adverbial like after Thursday is not acceptable, because it does not explicitly relate to now or $e_{\text {prev }}$, while since Thursday is acceptable because it does. Two days ago, while explicitly related to now, does not indicate a time that abuts now, and so it can not be used with the present perfect, whereas in the past two days can. (Two days before can be used with the past perfect syntactic form, but only for the past-of-past reading, and not the perfect.)

Indefinite adverbials If an adverbial does not fit the constraints on the reference times, then it cannot be used to modify the reference time. This does not necessarily mean that it cannot be used in a present perfect sentence, but if it is, then it cannot modify either of the two reference times set up by the perfect. There are some cases, as noted in Klein (1992), in which an adverbial such as on Sunday appears in a present-perfect sentence but does not specify a definite time location for the event:

(21) "Why is Chris in jail?" "He has worked on Sunday, and working on Sunday is strictly forbidden in this country."

This is one of those cases in which the adverbial does not anchor the event in time, but rather gives a specification on the type of timespan in which the event occurred. Here the adverbial would not take the usual meaning of the phrase on Sunday as being the most recent or upcoming Sunday, and it would not modify either of the perfect's reference times, but rather would be incorporated into the event at a lower structural level.

A similar constraint against definite reference is imposed on when-clauses that modify a perfect, for similar reasons. In order for a when-clause to felicitously modify an event carrying a perfect reading, the when-clause must indicate only a general type of eventuality (indefinite), as in (22), and not specify a particular actual event or time (definite), as in (23):

(22) a. I have driven in Toronto when it was snowing. (Norma Spejewski, p.c.)

b. I have been at the racetrack when a car overturned.

(23) a. ??I have driven in Toronto when they had that big blizzard.

b. ??I have been at the racetrack when Numero Uno died.

This covers what kinds of adverbs can be used with the perfect in general, based on semantic constraints. In the next section we will look at further constraints on adverbs, which rely on our pragmatic knowledge, either about event types or about particular situations.

\subsection{Adverbials and Pragmatics}

Using the present perfect with an adverbial seems to have the effect of predicating a frame adverbial over a presupposed event. This allows our world-knowledge- 
based expectations to come into play, and potentially to conflict with the adverbial information.

Recall that in the theory of temporal subordination, the reference time of an event is described as the time during which a speaker or hearer would expect an event to have occurred, given either linguistic or pragmatic cues. We typically have some knowledge about how long particular kinds of events take, and we can vary our interpretations of reference times accordingly. We also often have knowledge about when a given type of event is likely to occur, or how often it tends to be repeated. For instance, we may know that John typically walks his dog every morning, or that · Mary pays her Visa bill once a month. This kind of information also comes into play in the use of adverbials with the perfect. Given that we know that John walks his dog every day in the morning, we can say (24a) with today or this moming, but not with this evening, even if John actually walked her in the evening today, rather than in the morning. Similarly, we can say (24b) only with an adverb that is not smaller than our expectation time for the event (Katz \& Spejewski (1994)). The simple past lacks the presuppositional element and does not tap into our expectations in the same way.

(24) a. Wade has walked the dog \{today/this morning/??this evening\}.

b. Has Kay paid her bills \{this month/??this week\}?

Since we typically expect a person to die or be born once, but without any expectations of when that will be, any sentential adverbial modifying a particular birth or death or similar singular event in the perfect is odd:

(25) a. Our first child has been born \{??today/??this year/??recently .

b. The President has died \{??today/??this year/??recently\}.

c. Someone has killed the President \{??today/??this year/??recently\}.

d. My house has blown up \{??today .

This is explained by two features of the analysis: first, a reference time indicates an expected time for an event, and second, an adverbial must contain one of the available reference times. An adverbial that contains the expected timespan for the event is acceptable, but one that does not is pragmatically strange, even if it contains the actual location of the event. The use of reference times as a hearer's expectation, based partly on linguistic information and partly on pragmatic information, has both linguistic and pragmatic consequences for the interpretation of described events. The adverbials like yesterday were linguistically bad with the present perfect; the examples here are not as clearly bad as the earlier disallowed adverbials, but there is something rather strange about them, if we have the kind of pragmatic knowledge mentioned here. 


\subsection{States}

States in the perfect are subject to the same adverbial constraints as events in the perfect. The sentences in (26) show that up-to-now and including-now adverbials are fine with states, and (27) and (28) show that definite past adverbials do not work with states, nor do pragmatically-conflicting adverbials:

(26) a. Kent has lived in Prague \{for two years/since 1989\}.

b. The telephone has been ringing all morning.

c. I have been very happy $\{$ this weektoday\}.

(27) a. ??The telephone has been ringing yesterday/from 2 to 3 o'clock/an hour ago.

b. ??The president has been mortally ill this week.

(28) a. I have been with Frank when he lost his temper. [indefinite]

b. ??I have been with Frank ${ }_{i}$ when Kennedy died. [definite]

States in the perfect also seem to behave more like events than they do otherwise. Normally, states are considered to surround their reference times. Now if the state surrounds its reference time, and the reference time abuts now, then the state must also abut now. But in the perfect the state is not required to abut now:

(29) I have been in the White House (before).

Unlike the simple aspect, the perfect aspect introduces a state with a bounded beginning, and a possibly-bounded end. Because of this and the adverbial effects, I assume that in the perfect the state is contained in its reference time.

The interpretation of states in the perfect is not clear-cut, in that it involves implicatures. If an explicit frame adverbial is used, the state is contained within the adverbial time, and if an explicit durative adverbial is used, then the state is understood to hold throughout that time. If no adverbial is specified, then it is implied that the state is ended or of unknown status unknown at speech time. (30a) is fine with no adverbial, since Nixon's presidency is over, but (30b) is odd without modification, since Clinton is still president, in which case, the simple present is sufficient. Using a comples aspect implies that the simple is not sufficient. Given current history, the adverbial in (30a) can only be read as framing the event, and in (b) as the duration of the event. ( 3 la) with no adverbial makes no claim that anyone still is a member of the party, but when a durative adverbial is added, it does. If we use a state that is difficult to imagine ending, then its use in a perfect without adverbial modification is very strange, as with (32); for an intrinsic property of an individual, as in (32b), we also can't have it start after the individual came into being, although possibly at that point.

(30) a. Nixon has been president (since 1960). [frame]

b. Clinton has been president ??(since 1993). [durative] 
(31) a. Everybody has been a member of the Communist Party (for the last 5 years) [durative].

(32) a. John has liked ice cream ??\{since he was a baby\}).

b. Jackie has been Spanish ??\{??since 1990/??for 2 years/since the day she was born\}

\subsection{Negated Perfects}

The difference in interpretation between a negated present perfect and a negated simple past can also be explained by the way reference times are defined. As an example, consider (33):

(33) a. I haven't seen that movie.

b. I didn't see that movie.

Katz \& Spejewski (1994) propose that (a) implies that I still have the chance to see the movie, whereas (b) carries no such implication, and may even imply the reverse. This is accounted for by the perfect having two reference times, each of which is a pragmatic entity. First, I will assume a fairly simple interpretation of negated events: there is no event of the given type within the reference time. Now, suppose we have a simple past negation. This means we have some reference time which is strictly in the past of now, and the negation is interpreted as holding throughout the past reference time ((34a) below). But if we have a negated present perfect, we have two reference times. One abuts now, and this is the one introduced by the negated event, so the negation will hold throughout that reference time. However, there are two times being discussed here, and both are relevant for the interpretation. The perfect's reference time, which contains now, has not had negation claimed for this entire time. Since this reference time is one of our expectation times, it is still possible for the event to occur sometime within $R_{\text {now }}$, but only at a time which is after now.

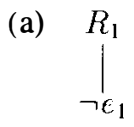

(b)

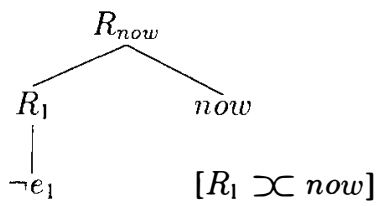

\section{Discourse Predictions of the Analysis}

The constraints on where to attach new events into a temporal discourse structure also hold for the perfect, and this allows us to predict what kinds of sequences we can have in a discourse subsequent to a perfect. Recall that the reference nodes along the right frontier are referred to as "open nodes", and that a new event can only 
be attached as the daughter of an open node. (Since now is not a reference node, the ordering between now and $R_{p}$ doesn't matter.) Suppose we have a discourse in which the most recently incorporated event was in the present perfect. We will then have just added two new reference nodes to the structure, and they will both be on the right frontier, since they will be descendents of a node on the right frontier. Leaving out any previous events, the structure would look like (35), with open nodes marked with "[+o]":

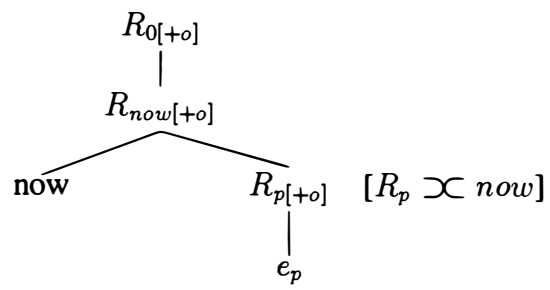

From this structure, we can predict what kinds of relations are possible for the next event introduced in the discourse. A new event's reference time must be the daughter of a currently-open node, and we can see the potential incorporation sites as indicated by the dashed lines:

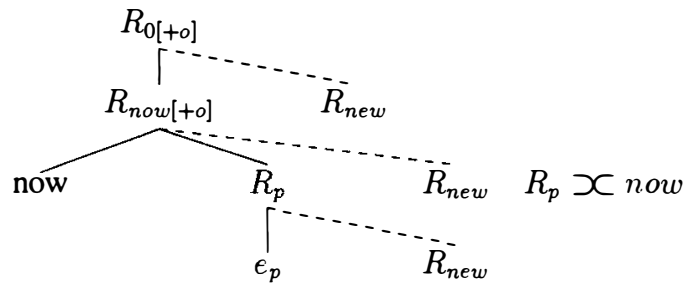

The interpretations for these different positions indicate how the new event is related to the present-perfect event. If the new reference time is a daughter of $R_{0}$ (or of any open reference node between $R_{0}$ and $R_{\text {now }}$ ), then the eventuality has been understood as being temporally independent from the eventuality that was introduced in the perfect; i.e. it is not subordinate to it, and it is also not subordinate to now. If the new reference time is inserted under $R_{\text {now }}$, then a special claim is being made: that the new event is subordinate to now; since I know of no other case of subordination to now, this event (probably) must be in the present perfect as well. Finally, if the new node is attached to $R_{p}$, then the new event is subordinate to the previous present perfect event, for instance as an event decomposition. An event which is in the present perfect must be attached at either $R_{\text {now }}$ or $R_{p}$, because it will be subordinate to now, and an event which is not in the present perfect must not be attached to $R_{\text {now }}$. It is possible for an event that is attached to $R_{p}$ not to be in the present perfect, as we see in (37d), because a reference node contained in $R_{p}$ need not abut now. Each of these readings are predicted by the general temporal 
subordination theory, and below we can see examples of each reading, with the attachment site given after each.

(37) a. The president has insisted on his innocence all along. The committee decided they needed proof instead. $\left[R_{0}\right]$

b. Jason has washed the dishes. He has put away the leftovers. [ $\left.R_{\text {now }}\right]$

c. Sandy has prepared a feast for dinner. She has made crepes and souffle. $\left[R_{p}\right]$

d. Sandy has prepared a feast for dinner. She made crepes and souffle. $\left[R_{p}\right]$

We can also see a case that is ruled out by the theory: a case in which the second event is subordinate to now but sequenced with the first event:

(38) ??Josh has picked up the book. (Then/Next) he has carried it to the table.

Here both the first and the second perfect must introduce a reference time that abuts now. If they both abut now, then these reference times must thus be in some kind of containment relation with each other. However, the interpretation that the events are sequenced can only be represented by having the reference times be sequenced with each other, and this gives an incompatible set of constraints. This means that no adverbial may modify the second in a sequence of present perfects if it indicates any kind of relation other than containment between the reference times. A pragmatically-understood sequencing is disallowed for similar reasons.

\section{Summary}

Interpreting the English perfect within the theory of temporal subordination accounts for a number of apparent idiosyncrasies of the English perfect as being instead predictable elements of a general theory of temporal relations in discourse. This analysis casts the perfect as a case of temporal subordination, where subordination forces the reference time of one event to be interpreted within the time introduced by another event or situation. For the present perfect, the event being introduced is subordinate to now, which means that the event is understood to occur within a timespan containing now, and for the past perfect the event occurs within a timespan introduced by a previous event in the discourse. The unique contribution of the English perfect is that the reference time containing the event must abut now or the previously-established time. This constraint appears to be missing in other languages, even when the perfect seems to require subordination, as with Spanish.

The interpretation of temporal frame adverbials as containing a reference time, along with the perfect's interpretation, account for many constraints on temporal modification of the perfect. A frame adverbial must contain the event and also either contain or abut now. Another facet of adverbial constraint is accounted for by the 
pragmatic nature of reference times as times during which events are expected to occur.

The theory of temporal subordination also predicts what kinds of discourse sequences can occur after a perfect, and what kinds of interpretations they can have, based on both linguistic and pragmatic factors.

Elements of other analyses of the perfect fall out directly from the analysis given here. For instance, the two different forms of the "extended-now" are manifest as the reference time of the event and the reference time for now; the idea of "current relevance" is inherent in the dependency structure between the event and now; and Klein's p-definiteness constraint falls out from the containment relation between the two reference times, and the Reichenbachian $\mathbf{E}, \mathbf{S}$, and $\mathbf{R}$ are present as $e, n o w$, and $R_{1}$.

\section{Endnote}

I wish to thank Greg Carlson, Graham Katz, my family, Chris Barker, Silvia Gennari, Montserrat Sanz, the Cliff group at Penn, and the SALT VI participants for helpful discussions on this material.

\section{References}

Bennett, Michael and Barbara Partee, 1978. Toward the Logic of Tense and Aspect in English. Indiana University Linguistics Club.

Binnick, Robert J., 1991. Time and the Verb: A Guide to Tense and Aspect. New York: Oxford University Press.

Comrie, Bernard, 1968. Aspect. Cambridge: Cambridge University Press.

Hinrichs, Erhardt, 1986. Temporal Anaphora in Discourses of English. Linguistics and Philosophy, 9, 63-82.

Kamp, Hans and Uwe Reyle, 1993. From Discourse to Logic. Dordrecht, The Netherlands: Kluwer.

Katz, Graham and Beverly Spejewski, 1994. Temporal Adverbials and the English Present Perfect. Annual Meeting of the LSA.

Klein, Wolfgang, 1992. The present perfect puzzle. Language 68,3, 525-552.

Lascarides, Alex and Nicholas Asher, 1991. Discourse Relations and Defeasible Knowledge. Proceedings of the 29th Meeting of the ACL.

Lascarides, Alex and Nicholas Asher, 1993. Temporal Interpretation, Discourse Relations and Commonsense Entailment. Linguistics and Philosophy, 16. 437-493.

McCawley, James D., 1973. Grammar and Meaning. Tokyo: Taishukan.

McCoard, Robert, 1978. The English Perfect: Tense-Choice and Pragmatic Inferences. Amsterdam: North-Holland. 
Michaelis, Laura, 1994. Temporal Anaphora and the Preterite-Perfect Contrast in English. Annual Meeting of the LSA.

Moens, Marc and Mark Steedman, 1988. Temporal Ontology and Temporal Reference. Computational Linguistics, 14, No. 2, 15-28.

Partee, Barbara, 1986. Nominal and Temporal Anaphora. Linguistics and Philosophy, 7 243-286.

Polanyi, Livia, 1988. A Formal Model of the Structure of Discourse. Journal of Pragmatics, 12, 601-638.

Reichenbach, H., 1947. Elements of Symbolic Logic. Berkeley, CA: University of California Press.

Ritchie, Graeme D., 1979. Temporal clauses in English. Theoretical Linguistics, 6, pp 89-115.

Spejewski, Beverly, 1994. Temporal Subordination in Discourse. Dissertation, University of Rochester.

Spejewski, Beverly and Greg N. Carlson, 1994. Modification of Event Relations. Proceedings from the 23rd Annual Conference of the North Eastern Linguistics Society, Ottawa, Canada, published by the Graduate Linguistics Students Association of University of Massachusetts.

Spejewski, Beverly and Greg N. Carlson, 1992. Reference Time Relations. Proceedings from the 1991 Eastern States Conference on Linguistics, Baltimore; The Ohio State University Press.

Twaddell, W.F., 1968. The English Verb Auxiliaries [2]. Providence, RI: Brown University Press.

Vlach, Frank, 1993. Temporal Adverbials, Tenses and the Perfect. Linguistics and Philosophy, 16, 231-283.

Webber, Bonnie, 1991. Structure and Ostension in the Interpretation of Discourse Deixis. Language and Cognitive Processes, 6, 107-135. 